\section{A CRISPR possibility for DMD}

\section{By Lauren Martz, Staff Writer}

CRISPR-based genome editing has filled the headlines since its discovery about two years ago for its ability to modify specific sequences in the genome with high accuracy, but its impact to date has been mainly as a research tool. A team from The University of Texas Southwestern Medical Center has now shown that the technology could have a therapeutic use in Duchenne muscular dystrophy, a disease involving mutations in the dystrophin gene. ${ }^{1}$

CRISPR (clustered, regularly interspaced short palindromic repeats) and DMD might be an obvious marriage of technology and disease because there is a direct link between resolving the mutations and improving symptoms in DMD. But the technology faces an uphill path to solve the key challenge of how to scale and deliver it in humans.

Eric Olson, principal investigator on the study, told SciBX, "I believe DMD is an ideal disease indication for CRISPR-mediated genomic editing because the molecular lesions responsible for the disease are well defined and there is an excellent animal model for testing." Olson is chairman and professor in the Department of Molecular Biology at UT Southwestern Medical Center.

The technique-dubbed CRISPR-Cas9-involves using single guide RNA (sgRNA) to direct the enzyme Cas9 to a desired location on the genome. Once Cas9 finds the right location, it induces a doublestranded break in the DNA, which triggers DNA repair and editing.

To date, the primary report of a disease application for the approach is a recent study by a Massachusetts Institute of Technology team that showed CRISPR could correct a mutation in fumarylacetoacetate hydrolase (Fah) and thereby improve symptoms in a mouse model of tyrosinemia type $1 ., 3$

Although Editas Medicine and CRISPR Therapeutics were founded to create therapeutics using CRISPR, neither company has yet disclosed any therapeutic programs.

Despite the early stage of the technology and the hurdles ahead, some researchers think a CRISPR-based product could ultimately be a more effective option than other more advanced DMD compounds in the clinic because-in contrast to those strategies-the approach corrects rather than bypasses the mutation.

"Antisense oligonucleotides modify the RNA to affect protein production, and therefore the effect is temporary. A real advantage of the CRISPR approach is that it is making a permanent modification to the cell's DNA. As long as the cell survives, it will continue to produce the corrected protein," said Charles Gersbach, an assistant professor of biomedical engineering at Duke University.
In DMD, dystrophin mutations disrupt production of the muscle fiber protein, which leads to wasting of skeletal, respiratory and cardiac muscles and reduces expected lifespan to about 25 years.

Although there are not yet any disease-modifying DMD therapies on the market, there are several in the clinic and three compounds at late stages of development: PTC Therapeutics Inc.'s Translarna ataluren, Prosensa Holding N.V.'s drisapersen and Sarepta Therapeutics Inc.'s eteplirsen.

Translarna is a small molecule that facilitates complete readthrough of proteins with missense mutations. The compound received conditional approval from the European Commission pending completion of a confirmatory Phase III trial. Drisapersen is an antisense oligonucleotide that induces skipping of exon 51 on the dystrophin gene and is now in Phase III testing. Eteplirsen is an antisense RNA that also induces exon 51 skipping. It is in Phase II testing.

Now, Olson and colleagues have tested CRISPR in a DMD model and shown that manipulating mouse zygotes from the model to affect the germline can help prevent and resolve DMD pathology.

The team used the $M d x$ model of DMD, which contains a nonsense point mutation in exon 23 of dystrophin. The researchers injected sgRNA targeting the mutant, Cas9 and a single-stranded oligonucleotide template to guide gene repair and correction into mouse $M d x$ zygotes and then implanted them into mothers.

The strategy created mosaic mice with different proportions of corrected dystrophin gene, ranging from $2 \%-100 \%$. However, when they analyzed four different muscle groups (quadriceps and soleus skeletal muscle, and cardiac and diaphragm), they found correction of a fraction of the genes was enough for a therapeutic benefit.

In a mouse with a $41 \%$ gene correction, the DMD phenotype was completely corrected, and in a mouse with a $17 \%$ correction, the disease was mostly corrected. Mice with the correction had better muscle strength than noncorrected $M d x$ mice. In addition, serum creatine kinase, a marker for DMD, was also decreased in corrected mice, and the level correlated with the degree of gene correction.

Next, the team looked
"The most relevant point of this study is not germline modification but that only a fraction of satellite cells needed gene correction to rescue the mutant phenotype. This might open the door for appropriate postnatal delivery methods in humans."

-Rodger Novak, CRISPR Therapeutics at the change in muscle fibers over time. In muscles from a mouse with about $40 \%$ gene correction at 3 weeks, there were no dystrophin-negative muscle fibers in skeletal muscle remaining at 9 weeks.

Together the findings suggested a selective advantage for the corrected cells and that CRISPR-Cas9 may have corrected satellite cells-skeletal muscle stem cells that give rise to new cells capable of dystrophin production. Indeed, when the researchers isolated and tested satellite cells, they confirmed that the mutation had been corrected.

"The authors use a therapeutic approach-germline editing - that is currently not acceptable in people," said Rodger Novak, founder and 
CEO of CRISPR Therapeutics. "The most relevant point of this study is not germline modification but that only a fraction of satellite cells needed gene correction to rescue the mutant phenotype. This might open the door for appropriate postnatal delivery methods in humans."

Because genomic editing in germline cells cannot be performed in humans both for ethical reasons and because the disease is typically diagnosed at a few years of age, the technique will first need to be performed in postnatal or adult cells to translate the findings to the clinic.

Olsen said that his team is now testing the efficacy of this approach in adult mice.

Data were published in Science.

\section{Heterogeneous advantage}

The hurdles for using CRISPR-Cas9 in DMD are similar to those that face the technology in most therapeutic areas. They include, in particular, the ability to deliver enough compound to achieve an effect in every cell-or at least in enough cells to produce a therapeutic effect.

However, the approach could have advantages over some of the drawbacks of other genetic approaches in DMD. Those include the need for different therapeutics for each causal mutation in dystrophin, the inability to alter large or complicated mutations and the fact that antisense molecules cannot effectively penetrate the cardiac muscle.

Gersbach told SciBX, "The exon-skipping approach involves targeting specific exons. This means that each therapy is only beneficial to patients with mutations that can be corrected by skipping those exons. The exon 51-skipping therapy in clinical trials, for example, only addresses $13 \%$ of the DMD population."

He added that one way to affect a larger population with the exonskipping approach is to target multiple exons. "We know that if you target exons $45-55$, you are able to cover about $62 \%$ of DMD patients," he said. "The problem is that each individual antisense oligonucleotide targets an individual exon, so skipping 10 exons really involves combining 10 different drugs."

Gersbach added, "Antisense molecules are expensive, you need to give a large amount of them in order to have an effect and you need to administer the molecules frequently."

For CRISPR, too, he said, "there are different mutations in different sets of patients, so different therapeutics would need to be tailored for different patient groups. However, the benefit of CRISPR is that whether you are deleting one or ten exons, it still involves just two cuts in the genome."

Jon Tinsley added, "One potential unique opportunity for this CRISPR technology would be if it could modify mutations that exon skipping couldn't, such as larger, more complex deletions." Tinsley is CSO for DMD at Summit Corp. plc.

In the short term, whereas methods for systemic delivery are under investigation, one option for DMD might be local delivery of CRISPR-Cas9 to affected muscles.

"One simpler treatment option that could still have great value in DMD would be to look at localized delivery of modified satellite cells- or AAV to selected muscles-to help prevent their degeneration and still add quality of life in the later stages of diseases." Adeno-associated viral (AAV) delivery is the use of viral vectors to insert DNA into a target cell.

Although CRISPR may help solve some of the issues specific to $\mathrm{DMD}$, Tinsley is not convinced that DMD will be the best therapeutic indication to help get the CRISPR-Cas9 system into the clinic. "One could envisage this technology is more appropriate for monogenic genetic diseases where the target organ is easier to manipulate, such as the liver, pancreas or blood where the delivery of ex vivo cells is already feasible," he said.

Ellen Welch, head of genetic disorders at PTC Therapeutics, agreed and added, "Duchenne is a very high bar given the challenges with delivery to all of the muscles. The authors mention that there are vectors capable of delivering the technology to tissues expressing dystrophin, but if it were that easy, we would probably already have a gene therapy for the disease." She added that disorders of the eye or liver that would allow more localized delivery would be good first steps for a CRISPR-based therapeutic.

A potential immediate indication for CRISPR could be the design of more DMD animal models that capture all the different mutations. "One immediate use of this technology would be to generate dystrophin mutations in wild-type animals specifically designated to generate bespoke models for testing new antisense exon-skipping drugs to currently untargeted dystrophin exons," said Tinsley. Because the CRISPR-Cas9 strategy has already been validated in nonhuman primates, the approach could also be used to design larger animal models of DMD to help test new therapeutics, he said.

Olson told SciBX that UT Southwestern Medical Center has filed a patent application covering this work and some additional new ideas and that the IP is available for licensing.

Martz, L. SciBX 7(38); doi:10.1038/scibx.2014.1115

Published online Oct. 2, 2014

\section{REFERENCES}

1. Long, C. et al. Science; published online Aug. 15, 2014; doi:10.1126/science. 1254445

Contact: Eric N. Olson, The University of Texas Southwestern Medical Center, Dallas, Texas

e-mail: eric.olson@utsouthwestern.edu

2. Yin, H. et al. Nat. Biotechnol. 32, 551-553 (2014)

3. Donner, A. SciBX 7(16); doi:10.1038/scibx.2014.447

\section{COMPANIES AND INSTITUTIONS MENTIONED}

CRISPR Therapeutics, Basel, Switzerland

Duke University, Durham, N.C.

Editas Medicine, Cambridge, Mass.

Massachusetts Institute of Technology, Cambridge, Mass Prosensa Holding N.V. (NASDAQ:RNA), Leiden, the Netherlands PTC Therapeutics Inc. (NASDAQ:PTCT), South Plainfield, N.J. Sarepta Therapeutics Inc. (NASDAQ:SRPT), Cambridge, Mass. Summit Corp. plc (LSE:SUMM), Abingdon, U.K.

The University of Texas Southwestern Medical Center, Dallas, Texas 